\title{
Knowledge based Adoption Behavior of Poultry Farmers in Different Agro-Climatic Zones of West Bengal, India
}

\author{
Arunasis Goswami and Sukanta Biswas* \\ Department of Veterinary \& A.H. Extension Education, W.B. University of Animal and \\ Fishery Sc. Kolkata, W.B., India \\ *Corresponding author
}

K e y w o r d s
Adoption,
Knowledge,
Livelihood, Poultry
Farmers, Agro-
climate etc

\section{A B S T R A C T}

Poultry farming is one of the vital assets of livelihood generation for pro-poor rural stakeholders in various agro-climatic Zone of West Bengal, India. In the study, one district from each agro climatic zone i.e. 06 districts were purposively selected. The total $1178 \mathrm{GP}$ was selected purposively from six districts and all villages under $61 \mathrm{Gram}$ Panchayets (i.e. $5 \%$ of 1178 GP) was covered randomly in which 524 no's of villages was taken for this study. From each village one poultry farmer i.e. 524 no's of Poultry owners were selected for the study. The data collected through pre-tested structured interview schedule with 35 Nos. of independent variables along with knowledge and Adoption Index as dependent variables and analyzed through various statistical methods. The study explored that, majority of Poultry owners were middle age group(31-40 years), married, Hindu by religion, High school literacy, low income group, Business and Labour were main occupation and took self-decision for poultry farming in all zones. The majority of selected Poultry owners have medium level of knowledge and adoption about improved farming practices in all Zones. Poultry farming should be encouraged in all ACZ along with training of farmers for improved A.H. practices is to be taken due consideration while formulating planning.

\section{Introduction}

Poultry farming plays a pivotal role in livelihood generation of pro-poor stakeholders in various agro-climatic Zone of West Bengal, India. Realizing the present day challenge, like Poverty, malnutrition, livelihood insecurity and environmental crisis, scientific orientation for empowering rural Poultry farmers through knowledge based adoption practices are extremely needed under various agro-climatic zones of Eastern
India i.e. West Bengal. To develop sustainable vis-à-vis profitable Poultry farming practices and for empowering these farming communities a vivid study was promulgated in selected Poultry farmers under 06 Agro-climatic Zones of West Bengal, India.

\section{Materials and Methods}

In present study, one district from each agro climatic zone i.e. 06 districts were 
purposively selected from the state of W.B. The total 1178 GP was selected purposively from six districts and all villages under 61 Gram Panchayets (i.e. 5\% of 1178 GP) was covered randomly in which 524 no's of villages was taken for this study. From each village one poultry farmer i.e. 524 no's of Poultry owners were selected for the study. The field investigation was carried out with the help of pre-tested structured interview schedule constructed for the study. Total, Thirty five (35) no's of independent variables were selected to assess the adoption behavior of selected Poultry owners in various Agro climatic zone of W.B, India. The independent variables were measured with readily available scales and few scales were also developed for the study. Adoption of selected improved Poultry farming practices was dependent variable which was measured by adoption index method (Das Gupta, 1968). The collected data was computed and analyzed by various statistical methods for better interpretation of the study.

\section{Results and Discussion}

The analytical study of the table- 1 represents that, the adoption index in IAHP of selected Poultry owners in New alluvial Zone (NAZ) were positively and significantly correlated with variables like marital status, education, family education status, house type, material possession, attitude towards income generation, mass media, communication sources, risk orientation, social participation, at $1 \%$ level whereas with caste, personal Cosmo politeness, attitude towards employment, knowledge in poultry at $5 \%$ level of significance. In Old Alluvial zone (OAZ), adoption index in IAHP of selected poultry farmers had positive significant correlation with variables such as- other income sources, communication skill at 5\% level and with knowledge on Goatery at $1 \%$ level of significance. In Terrain zone, adoption index in IAHP were positively and significantly correlated with variables likePathology center, family type, Mass media, communication sources, Marketing orientation at $1 \%$ level, whereas with personal localiteness, Cosmo politeness, attitude in productivity, income generation at 5\% level of significance. In Hill zone, adoption index in IAHP were positively and significantly correlated with the variables like- Marital status, pathology center, economic motivation, attitude towards productivity at $5 \%$ level whereas with mass media, personal Cosmo politeness, localiteness, communication sources and skill, attitude towards income generation at $1 \%$ level of significance. In Red laterite zone (RLZ), adoption index have positive significant correlation with the variables like-marital status, family type, family size, mass media, personal Cosmo politeness, localiteness, communication sources at $1 \%$ level whereas with marketing orientation, family education status at 5\% level of significance. Finally, the adoption index in IAHP of selected poultry owners in Coastal zone were positively and significantly correlated with variables likePathology center, caste, family education status, knowledge in poultry farming, attitude in productivity, Marketing orientation, risk orientation, at $1 \%$ level whereas with Mass media at $5 \%$ level of significance under study area.

The following previous observations will support some where the present study to get better understanding. Sohal and Tyagi (1978) reported that family size had no significant association with knowledge in urban area. Chung (1986) also made similar reporting for economic status and positive relationship was reported by Chandra (1979) and Hazarika (1983). Sekhan (1970) and Sundaraswami et al., (1978) had made positive relation with utilization of mass media. Sarkar (1981), Sayeedi (1983) and Dana et al., (1998) found 
significant relation of family educational status with adoption of Poultry innovations. Daipuria et al., (2001) reported that age, education, house type, social participation, risk orientation, mass media, marketing orientation, knowledge were significantly associated with adoption of poultry farming practices.

The result of Path analysis for selected Poultry farmers represents the direct and indirect effects of the 44 selected exogenous variables on Adoption index of all zones of West Bengal. The data revealed that- Mass media (0.643) has largest direct effect on adoption index of poultry farmers followed in 05 no's of descending order variables such aspersonal localiteness (0.549), personal Cosmo politeness (0.436), knowledge in GFC (0.260), Education (0.170), attitude in employment (0.169) had positive direct effect whereas-knowledge in piggery (-0.002), communication skill (-0.012), economic motivation(-0.018), occupation (-0.039), religion(-0.049) had negative direct effect on adoption index of poultry farmers of all zones of West Bengal.

As regards, total indirect effect it was revealed from the table 2 that- communication sources (1.321), pathology centre(0.386), Knowledge in poultry farming (0.281), social participation (0.242) and knowledge in colostrum's feeding (0.206) were the first five factors which exerts largest indirect effects on adoption index in poultry farming practices.

The data also explored that out of 44 exogenous variables, maximum variables had their largest indirect effects through personal localiteness, personal Cosmo politeness, mass media, communication sources, knowledge in deworming, Education, attitude in productivity, employment and income generation, social participation, knowledge in poultry farming, family education status, total income, family type, knowledge in concentrate feeding, vaccination and other income sources etc. The residual effect has been found to be 0.63 or in a way, $63 \%$ of the total variability has been left unexplained.

Analytical study suggest that Mass media sources, personal localitenes, cosmopoliteness and communication sources not only exerts largest direct effects on adoption index in poultry farming, but also it influences indirectly in association with a large number of variables which perform their role through this factors. So, these factors have come out to be a key element which directly and indirectly promotes adoption of improved poultry farming practices in zone wise stakeholder's entrepreneurship development. Roy (2006) studied about the adoption behaviour of Kuroilar farmers in sundarbans and found the same results. Jha and shakwat (1972) observed in adoption practices that age has got a significant association.

Hussain (1968) reported that income of the respondent was not significantly associated with the adoption of improved animal husbandry practices. Tripathy and Jyoti (1971) suggested the significant association of family education with the adoption behaviour of the farmers. Goswami (2000) found that mass media and personal cosmopolite had positive and significant association with the improved A.H. practices.

The table 3 depicted that, mean value of poultry farmers under Zone-1 was significantly higher in relation to adoption of Vaccination, feeding of Concentrate and composite adoption practices. But the mean score of Poultry farmers under Zone-5 was significantly higher in relation to adoption of Deworming Practices. 
Table.1 Relationship between Selected Independent \& Adoption Index of Poultry Farmers in Various Agro-Climatic Zones of W.B

\begin{tabular}{|c|c|c|c|c|c|c|}
\hline \multirow[t]{2}{*}{ Variables } & Burdwan & Howrah & Coochbehar & Darjeeling & Purulia & S24pgs \\
\hline & NA Zone & OA Zone & TERAI Zone & HILL Zone & RL Zone & CL Zone \\
\hline Sex & -0.034 & 0.022 & 0.082 & -0.001 & 0.033 & -0.134 \\
\hline Age & 0.091 & 0.136 & -0.157 & -0.074 & -0.149 & -0.007 \\
\hline Religion & -0.237 & 0 & -0.04 & -0.107 & 0.227 & 0.058 \\
\hline Marital Status & 0.242 & -0.088 & 0.203 & 0.221 & 0.321 & 0.073 \\
\hline Income & -0.037 & -0.222 & -0.251 & 0.02 & -0.169 & -0.204 \\
\hline Oth Income & 0.111 & 0.244 & 0.015 & 0.041 & -0.028 & 0.279 \\
\hline Tot Income & 0.098 & -0.021 & 0.012 & 0.036 & -0.073 & 0.073 \\
\hline Pathology Centre & 0.043 & -0.048 & 0.511 & 0.249 & 0.157 & 0.342 \\
\hline Caste & 0.215 & -0.116 & 0.213 & 0.206 & -0.206 & 0.25 \\
\hline Milk Productivity & 0.208 & 0.00 & 0.317 & 0.00 & 0.293 & 0.333 \\
\hline Occupation & -0.086 & 0.00 & -0.337 & 0.00 & -0.113 & -0.237 \\
\hline Education & 0.456 & 0.00 & 0.004 & 0.00 & 0.119 & 0.122 \\
\hline Fam. Edu Stat & 0.598 & 0.00 & 0.099 & 0.00 & 0.258 & 0.412 \\
\hline Fam_Type & 0.109 & 0.00 & 0.633 & 0.00 & 0.617 & 0.169 \\
\hline Fam_Size & 0.094 & 0.00 & 0.241 & 0.00 & 0.429 & 0.054 \\
\hline Land & 0.153 & 0.00 & -0.221 & 0.00 & -0.009 & -0.165 \\
\hline House & 0.257 & 0.00 & 0.107 & 0.00 & 0.058 & 0.005 \\
\hline Farm Power & -0.002 & 0.00 & -0.268 & 0.00 & -0.273 & -0.127 \\
\hline Knw_Col & 0.312 & 0.137 & 0.314 & 0.005 & 0.011 & 0.316 \\
\hline Knw_Goat & 0.012 & 0.327 & -0.052 & -0.246 & -0.116 & 0.03 \\
\hline Knw_Pig & 0.027 & -0.124 & -0.288 & -0.277 & 0.023 & -0.161 \\
\hline Knw_Duck & 0.032 & -0.064 & -0.411 & -0.056 & -0.135 & -0.239 \\
\hline Knw Poultry & 0.2 & 0.116 & 0.201 & 0.005 & 0.304 & 0.298 \\
\hline Knw_Milk & 0.264 & -0.172 & 0.125 & -0.092 & 0.233 & 0.17 \\
\hline Att. Employment & 0.19 & -0.174 & -0.069 & -0.006 & -0.053 & -0.068 \\
\hline Att Income & 0.334 & -0.036 & 0.294 & 0.284 & 0.152 & 0.187 \\
\hline Mat Possession & 0.388 & 0.027 & 0.105 & -0.011 & 0.12 & 0.043 \\
\hline Economic Motiv. & -0.177 & 5.556 & -0.204 & 0.239 & -0.224 & -0.011 \\
\hline Attitude & 0.281 & 0.006 & 0.347 & 0.014 & -0.057 & 0.315 \\
\hline Knw_AI & -0.055 & -0.138 & 0.414 & 0.117 & -0.136 & 0.268 \\
\hline Knw_VAC & -0.117 & 0.192 & -0.08 & -0.105 & 0.095 & -0.22 \\
\hline Knw_DE & -0.243 & -0.392 & -0.219 & -0.321 & -0.037 & -0.187 \\
\hline Knw_GFC & 0.001 & 0.28 & -0.361 & -0.24 & 0.087 & -0.32 \\
\hline Knw_GFF & -0.038 & -0.258 & -0.153 & -0.036 & 0.16 & -0.186 \\
\hline Knw_CF & -0.123 & 0.202 & -0.343 & -0.377 & 0.016 & -0.251 \\
\hline Att. Productivity & 0.437 & -0.066 & 0.284 & 0.277 & -0.067 & 0.3 \\
\hline Mass Media & 0.274 & 0.182 & 0.422 & 0.421 & 0.586 & 0.23 \\
\hline Per Cosmopolite & 0.201 & -0.037 & 0.294 & 0.346 & 0.341 & 0.1 \\
\hline Per Localite & 0.101 & -0.089 & 0.332 & 0.469 & 0.375 & -0.15 \\
\hline Communication & 0.39 & 0.005 & 0.453 & 0.44 & 0.584 & 0.078 \\
\hline Com Skill & 0.116 & 0.281 & 0.17 & 0.383 & 0.232 & -0.014 \\
\hline Marketing Orient. & 0.135 & -0.148 & 0.371 & 0.17 & 0.279 & 0.554 \\
\hline Risk Orientation & 0.235 & 0.095 & 0.112 & 0.084 & 0.173 & 0.227 \\
\hline Social Participation & 0.225 & 0.18 & -0.112 & -0.016 & 0.145 & -0.087 \\
\hline
\end{tabular}

N.B. Bold values are significant at $5 \%$ level \& Bold and italics value are significant at $1 \%$ level 
Table.2 Path coefficient showing direct \& indirect effects of selected poultry farmers on adoption index in all (06) zones of W.B

\begin{tabular}{|c|c|c|c|c|c|c|c|c|}
\hline \multirow{2}{*}{$\begin{array}{l}\text { Independent } \\
\text { Variables }\end{array}$} & \multirow{2}{*}{$\begin{array}{l}\text { Direct } \\
\text { effect }\end{array}$} & \multirow[t]{2}{*}{ Rank } & \multirow{2}{*}{$\begin{array}{c}\text { Total indirect } \\
\text { effect }\end{array}$} & \multirow[t]{2}{*}{ Rank } & \multicolumn{4}{|c|}{ Substantial Indirect Effect (Via) } \\
\hline & & & & & Var. No & $1^{\text {st }}$ & Var. No & $2^{\text {nd }}$ \\
\hline $1 \mathrm{Sex}$ & -0.058 & 33 & -0.043 & 25 & 38 & 0.025 & 26 & 0.024 \\
\hline 2 Age & 0.064 & 15 & 0.020 & 20 & 31 & 0.069 & 40 & 0.066 \\
\hline 3 Relig & -0.049 & 32 & -0.084 & 30 & 39 & 0.084 & 25 & 0.069 \\
\hline 4 Mar_Stat & 0.094 & 13 & -0.049 & 26 & 37 & 0.126 & 39 & 0.061 \\
\hline 5 Incom & -0.194 & 41 & -0.041 & 24 & 25 & 0.037 & 7 & 0.035 \\
\hline 6 Oth_Inc & -0.092 & 35 & 0.062 & 15 & 40 & 0.115 & 12 & 0.052 \\
\hline 7 Tot_Inc & 0.051 & 17 & -0.175 & 38 & 24 & 0.042 & 40 & 0.042 \\
\hline 8 Path_Cen & 0.081 & 14 & 0.386 & 02 & 31 & 0.201 & 40 & 0.112 \\
\hline 9 Caste & 0.010 & 25 & -0.072 & 28 & 39 & 0.049 & 38 & 0.044 \\
\hline 10 Milk_Pro & 0.145 & 08 & -0.050 & 27 & 39 & 0.057 & 36 & 0.040 \\
\hline 11 Occup & -0.039 & 31 & -0.150 & 37 & 38 & 0.070 & 12 & 0.042 \\
\hline 12 Educatio & 0.170 & 05 & 0.028 & 19 & 37 & 0.039 & 13 & 0.039 \\
\hline 13 Fam_Edu & 0.120 & 11 & 0.073 & 12 & 39 & 0.074 & 12 & 0.055 \\
\hline 14 Fam_Type & 0.105 & 12 & 0.045 & 17 & 38 & 0.111 & 37 & 0.084 \\
\hline 15 Fam_Sz & 0.032 & 20 & 0.035 & 18 & 38 & 0.072 & 14 & 0.071 \\
\hline 16 Land & 0.001 & 27 & -0.007 & 21 & 12 & 0.068 & 39 & 0.058 \\
\hline 17 House & 0.012 & 24 & 0.192 & 06 & 38 & 0.078 & 31 & 0.054 \\
\hline 18 Farm_Pwr & 0.050 & 18 & 0.092 & 10 & 40 & 0.156 & 31 & 0.071 \\
\hline 19 Mat_Poss & -0.093 & 36 & 0.121 & 09 & 12 & 0.062 & 40 & 0.043 \\
\hline 20 Eco_Moti & -0.018 & 30 & -0.098 & 31 & 39 & 0.116 & 25 & 0.067 \\
\hline 21 Attitude & 0.002 & 26 & 0.070 & 14 & 33 & 0.046 & 36 & 0.039 \\
\hline 22 Knw_Ai & 0.019 & 23 & 0.081 & 11 & 38 & 0.067 & 31 & 0.060 \\
\hline 23 Knw_Vac & 0.029 & 21 & -0.269 & 40 & 39 & 0.157 & 25 & 0.154 \\
\hline 24 Knw_De & -0.200 & 42 & -0.139 & 35 & 25 & 0.142 & 39 & 0.087 \\
\hline 25 Knw_Gfc & 0.260 & 04 & -0.530 & 43 & 39 & 0.178 & 37 & 0.044 \\
\hline 26 Knw_Gff & -0.104 & 37 & -0.032 & 23 & 39 & 0.118 & 25 & 0.115 \\
\hline 27 Knw_Cf & -0.118 & 39 & -0.148 & 36 & 25 & 0.169 & 39 & 0.093 \\
\hline 28 Knw_Col & -0.062 & 34 & 0.206 & 05 & 33 & 0.064 & 38 & 0.060 \\
\hline 29 Knw_Goat & 0.023 & 22 & -0.100 & 32 & 39 & 0.076 & 33 & 0.054 \\
\hline 30 Knw_Pig & -0.002 & 28 & -0.121 & 33 & 25 & 0.121 & 40 & 0.038 \\
\hline 31 Knw_Duck & -0.272 & 43 & -0.235 & 39 & 39 & 0.218 & 25 & 0.187 \\
\hline 32 Knw_Poul & 0.140 & 09 & 0.281 & 03 & 31 & 0.076 & 33 & 0.053 \\
\hline 33 Knw_Milk & 0.135 & 10 & 0.071 & 13 & 32 & 0.055 & 40 & 0.038 \\
\hline 34 Att_ĒEmpl & 0.169 & 06 & -0.125 & 34 & 38 & 0.057 & 32 & 0.024 \\
\hline 35 Att_Inc & -0.127 & 40 & 0.143 & 08 & 37 & 0.127 & 39 & 0.124 \\
\hline 36 Att_Prod & 0.115 & 38 & -0.027 & 22 & 10 & 0.050 & 24 & 0.047 \\
\hline 37 Mass_Me & 0.643 & 01 & -0.337 & 42 & 39 & 0.236 & 38 & 0.188 \\
\hline 38 P_Cos & 0.436 & 03 & -0.311 & 41 & 39 & 0.279 & 37 & 0.278 \\
\hline 39 P_Loc & 0.549 & $\mathbf{0 2}$ & -0.654 & 44 & 37 & 0.276 & 38 & 0.221 \\
\hline 40 Commun & -1.199 & 44 & 1.321 & 01 & 37 & 0.480 & 39 & 0.450 \\
\hline 41 Com_Skil & -0.012 & 29 & -0.076 & 29 & 37 & 0.244 & 39 & 0.243 \\
\hline 42 Market_O & 0.062 & 16 & 0.189 & 07 & 37 & 0.082 & 31 & 0.046 \\
\hline 43 Risk_O & 0.048 & 19 & 0.055 & 16 & 38 & 0.095 & 37 & 0.093 \\
\hline 44 Soc_Part & 0.152 & 07 & 0.242 & 04 & 37 & 0.115 & 31 & 0.073 \\
\hline
\end{tabular}

Residual Effect=0.630777 
Table.3 Zone wise comparison by Chi-Square (KW) test of selected poultry owners in 06 agroclimatic zone of West Bengal

\begin{tabular}{|l|c|c|c|c|c|c|c|}
\hline Zone & Ad.Vacc & Ad.Dewor & AD.CF & Ad. Colost & Ad Burdiz & Ad.Ureastrw & Adoption \\
\hline 1.OAZ & 3.85 & 3.55 & 3.20 & 3.36 & 3.21 & 2.23 & 3.33 \\
\hline 2.NAZ & 3.52 & 3.65 & 0.67 & 0.67 & 0.20 & 0.04 & 1.19 \\
\hline 3.TERAI & 3.46 & 3.74 & 0.86 & 1.02 & 0.23 & 0.00 & 1.42 \\
\hline 4.HILL & 2.75 & 3.07 & 0.60 & 1.31 & 0.12 & 0.00 & 1.16 \\
\hline 5.RL & 3.45 & 3.84 & 1.55 & 2.42 & 0.92 & 0.14 & 2.08 \\
\hline 6.COSTAL & 3.42 & 3.55 & 0.69 & 1.14 & 0.19 & 0.02 & 1.38 \\
\hline Chi Squre & 16.34 & 12.41 & 133.75 & 110.28 & 250.10 & 266.44 & 237.78 \\
\hline Sig. & $\mathbf{0 . 0 1}$ & $\mathbf{0 . 0 3}$ & $\mathbf{0 . 0 0}$ & $\mathbf{0 . 0 0}$ & $\mathbf{0 . 0 0}$ & $\mathbf{0 . 0 0}$ & $\mathbf{0 . 0 0}$ \\
\hline
\end{tabular}

Table.4 Stepwise Multiple Regression analysis between some selected Independent \& Dependent variables (Adoption) of Sample Poultry Farmers

\begin{tabular}{|c|c|c|c|c|c|}
\hline \multirow[t]{2}{*}{ Variables } & \multicolumn{2}{|c|}{ Unstand. Coefficients } & \multirow{2}{*}{$\begin{array}{l}\text { Stand. } \\
\text { Coefficients } \\
\text { Beta }\end{array}$} & \multirow[t]{2}{*}{$\mathbf{t}$} & \multirow[t]{2}{*}{ Sig. } \\
\hline & B & Std. Error & & & \\
\hline (Constant) & 0.301 & 0.248 & & 1.214 & 0.225 \\
\hline Knw_Duck & -0.026 & 0.005 & -0.334 & -5.630 & 0.000 \\
\hline Mass Media & 0.067 & 0.011 & 0.187 & 5.875 & 0.000 \\
\hline Knw Poultry & 0.040 & 0.007 & 0.213 & 5.669 & 0.000 \\
\hline Social Participation & 0.170 & 0.039 & 0.166 & 4.415 & 0.000 \\
\hline Income & -0.109 & 0.030 & -0.125 & -3.678 & 0.000 \\
\hline Education & 0.073 & 0.020 & 0.134 & 3.665 & 0.000 \\
\hline Occupation & -0.068 & 0.020 & -0.112 & -3.352 & 0.001 \\
\hline Knw_Gff & -0.044 & 0.015 & -0.111 & -2.996 & 0.003 \\
\hline Knw_Gfc & 0.022 & 0.004 & 0.257 & 5.013 & 0.000 \\
\hline Knw_De & -0.063 & 0.017 & -0.154 & -3.790 & 0.000 \\
\hline Fam. Edu Stat & 0.076 & 0.029 & 0.093 & 2.642 & 0.008 \\
\hline Fam_Type & 0.270 & 0.076 & 0.116 & 3.548 & 0.000 \\
\hline Milk Productivity & 0.031 & 0.011 & 0.102 & 2.894 & 0.004 \\
\hline Pathology Centre & 0.247 & 0.116 & 0.107 & 2.134 & 0.033 \\
\hline \multicolumn{6}{|l|}{ Model Summary } \\
\hline $\mathbf{R}$ & R Square & Adjusted R Sq & SE (Est) & & \\
\hline 0.743 & 0.552 & 0.539 & 0.672 & & \\
\hline
\end{tabular}

Note: * $\mathrm{P}>0.05$ level; $* * \mathrm{P}>0.01$ level; $\mathrm{F}$ entry Probability $=0.05 ; \mathrm{F}$ removal probability $=0.10$ 
Table.5 Utilization of different information sources in adoption of IAHP by selected Poultry farmers in various Agro-climatic Zones of W.B

\begin{tabular}{|c|c|c|c|}
\hline \multicolumn{4}{|c|}{ Sources of Information } \\
\hline ACZ of W.B. & $\begin{array}{l}\text { Mass } \\
\text { Media }\end{array}$ & $\begin{array}{c}\text { Personal Cosmo } \\
\text { politeness }\end{array}$ & $\begin{array}{l}\text { Personal } \\
\text { localitness }\end{array}$ \\
\hline Hill Zone(84) \% \& Rank & $44.05(\mathrm{~V})$ & $52.38(\mathrm{~V})$ & 65.48(II) \\
\hline Coastal Zone(108) \% \& Rank & $38.89(\mathrm{VI})$ & $52.78(\mathrm{IV})$ & $66.67(I)$ \\
\hline RL Zone(68) \% \& Rank & $44.12(\mathrm{IV})$ & 52.94 (III) & 61.76 (III) \\
\hline NA Zone(132) \% \& Rank & $56.82($ III) & $60.61(\mathrm{I})$ & $43.94(V)$ \\
\hline OA Zone(75) \% \& Rank & 60.00 (II) & $50.67(\mathrm{VI})$ & $36.00 \mathrm{VI}$ \\
\hline Terrain Zone(57) \% \& Rank & $73.68(I)$ & 54.39 (II) & 45.61 IV \\
\hline
\end{tabular}

Table.6 Knowledge\& Adoption level of Poultry owners about improved A.H. practices in various Agro-climatic zones (06) of W.B., India

\begin{tabular}{|c|c|c|c|c|}
\hline AC Zone & Level & Statistical parameter & Knowledge level & Adoption level \\
\hline \multirow{3}{*}{$\begin{array}{l}\text { Burdwn } \\
\text { (NAZ) }\end{array}$} & Low & $<($ Mean-SD) & 18.70 & 14.70 \\
\hline & Medium & (Mean - SD) to (Mean+SD) & 62.80 & 73.40 \\
\hline & High & $>($ Mean+SD $)$ & 18.50 & 11.90 \\
\hline \multirow{3}{*}{$\begin{array}{l}\text { Howrah } \\
\text { (OAZ) }\end{array}$} & Low & $<($ Mean-SD) & 7.10 & 14.30 \\
\hline & Medium & $($ Mean - SD) to $($ Mean + SD) & 74.90 & 72.50 \\
\hline & High & $<($ Mean-SD) & 18.00 & 13.20 \\
\hline \multirow{3}{*}{$\begin{array}{l}\text { Purulia } \\
\text { (RLZ) }\end{array}$} & Low & $<($ Mean-SD) & 10.50 & 21.10 \\
\hline & Medium & $($ Mean - SD) to $($ Mean $+S D)$ & 71.70 & 64.80 \\
\hline & High & $<($ Mean-SD $)$ & 17.80 & 14.10 \\
\hline \multirow{3}{*}{$\begin{array}{l}\text { Cooch-behar } \\
\text { (TRZ) }\end{array}$} & Low & $<($ Mean-SD) & 16.20 & 13.20 \\
\hline & Medium & $($ Mean - SD) to $($ Mean + SD) & 70.60 & 64.70 \\
\hline & High & $<($ Mean-SD) & 13.20 & 22.10 \\
\hline \multirow{3}{*}{$\begin{array}{l}\text { Darjeeling } \\
\text { (HLZ) }\end{array}$} & Low & $<($ Mean-SD) & 13.60 & 14.40 \\
\hline & Medium & $($ Mean - SD) to $($ Mean + SD) & 59.90 & 68.10 \\
\hline & High & $<($ Mean-SD $)$ & 26.50 & 17.50 \\
\hline \multirow{3}{*}{$\begin{array}{l}\text { South } 24 \text { Pgs } \\
\text { (CSZ) }\end{array}$} & Low & $<($ Mean-SD) & 20.40 & 11.10 \\
\hline & Medium & $($ Mean - SD) to $($ Mean $+S D)$ & 64.80 & 77.80 \\
\hline & High & $<($ Mean-SD $)$ & 14.80 & 11.10 \\
\hline
\end{tabular}

The findings depicted above may be supported by previous research findings as follows. Kunzru (1987), Sivnarayana et al., (1995) and Goswami (2000) worked on same characteristics and found more or less similar observations. Goswami (2007) found that education of livestock owner has got its impact on improved poultry farming practices. Singh (1982) and Nataraju \& Channegowda (1984) established that mass media source had significant association with the adoption of improved poultry farming practices. Roy (2006) also observed that social participation had significant association with adoption of improved poultry farming practices. 
The table 4 depicted that $55.00 \%$ of the variation in adoption index in IAHP of sample poultry farmers was due to combined effect of the selected independent variables contributed in the analysis. The result in the table 5 indicated that the variables like- Mass media, knowledge in Poultry, social participation, education, family education status, family type showed positive and highly significant $(\mathrm{P}<0.01)$ contribution while pathology center was contributing significantly at 5\% level of significance. But the variable like knowledge in Duckery, income source, occupation, knowledge in GFF and deworming showed negative and highly significant contribution $(\mathrm{P}<0.01)$. The other variables were excluded as redundant in improving adoption index in IAHP of the selected sample poultry farmers under all zones of West Bengal. Roy (2006) studied about the adoption behavior of Kuroilar farmers in Sundarbans and found same results. Tripathy and Jyoti (1971) suggested significant association of family education with adoption behaviour of the farmers. Goswami (2000) found that mass media and personal cosmopolite had positive-significant association with improved A.H. practices.

The Tabular value explored that, maximum selected poultry farmers in Terrain Zone (73.68\%) utilized several mass media as an information sources regarding adoption of improved poultry farming practices, followed by the respondents of OAZ $(60.00 \%)$, NAZ (56.82\%), RLZ (44.12\%), HLZ (44.05\%) and CLZ (38.89\%) were utilize various mass media as information sources in adoption of IAHP rank wise in descending order. Similarly, considering personal cosmopolite information sources utilization, higher no. of selected poultry farmers in New alluvial zone (Rank-I) used various channel regarding adoption of improved poultry farming practices, whereas selected respondents of TZ(rank-II), RLZ(rank-III), CLZ(rank-IV),
HLZ(rank-V), and OAZ(rank-VI) were sequentially utilize several personal cosmopolite sources for adoption of improved poultry farming practices. Simultaneously, majority of selected poultry owners in coastal zone $(66.67 \%)$ used Personnel Localite Sources in adoption of improved poultry farming practices, as well as selected respondents of HLZ(65.48\%), RLZ(61.76\%), TRZ(45.61\%), NAZ(43.94\%), and OAZ $(36.00 \%)$ were chronologically utilize personal localite channels in descending order as information sources for adoption of improved poultry farming practices in the study area.

The Table 6 revealed that level of knowledge and adoption of selected Poultry owners about improved farming practices in various Agroclimatic zones of West Bengal. The tabular fact explored that, $60-75 \%$ of Poultry owners have medium knowledge level and $65-78 \%$ have medium adoption level on scientific poultry farming practices and only scanty $\%$ have low and high level of knowledge and adoption index on recommended practices in all $06 \mathrm{ACZ}$ of West Bengal. So, the analytical study concluded that majority of selected Poultry entrepreneurs have medium knowledge and adoption level about improved farming practices in all $06 \mathrm{ACZ}$ of West Bengal, which is very much indicative in relation to future animal Husbandry development strategy as well as planning of W.B.

In conclusion the analytical study revealed that among all independent variables such asMass media sources, personal localitenes, cosmopoliteness and communication sources, attitude \& knowledge level were the key elements which directly and indirectly helped to improve the adoption index of Poultry farmers for successful entrepreneurship development in all zones of W.B. India. The important variables such as-Mass media, 
knowledge in Poultry, social participation, education, family education status, family type were the key variables to measure adoption index of Poultry owners in all ACZ of W.B. Majority of Poultry owners in Terrain Zone utilized several mass media as an information sources, whereas in New alluvial zone, majority used personal cosmopolite information sources and in Coastal zone, majority used Personnel Localite Sources for adoption of improved poultry farming practices. The majority of selected entrepreneurs had medium Knowledge and adoption in improved Poultry farming practices in all Zones of W.B.

On the basis of the analytical study, it was recommended that, to formulate any development planning, emphasis should be given on all communities, income, literacy, Marketing orientation, Attitude, knowledge level, adoption rate of improved technologies, mass media exposure, social participation, personal Cosmo politeness and personal localiteness are to be considered for substantial empowerment of stakeholders through Poultry enterprises. The basic parameters are more or less same and only degree and magnitude of parameters will vary depending upon different ACZ, for which experts are to be consulted during planning. Poultry farming should be encouraged for all types of people in all AC zones- specially, New Alluvial, Hill, Terrain and Coastal Zones are to be more emphasized for this farming practices.

\section{References}

Chandra, K. (1979). Differential adoption of dairy innovations by the farmers of ICDP, Karnal. Ph.D. Thesis (Unpublished), P.U., Chandigarh.

Chug, D. S. (1986). Suitability of dairy farming technology and factors affecting knowledge and adoption.
M.Sc. Thesis submitted to the G. B. Pant University of Agril \& Technology, Pantnagar.

Daipuria, P. R. (2001) Knowledge and adoption of Agricultural Technologies in Maratha Wada. Indian Res. J. Ext. Edu. 7(1) January.

Dana, S.S. Khandekar, N, Sharma, R. P. and Sinha, S. P. (1998). Factors affecting adoption of commercial poultry production technologies. Indian Jr. of animal Res. 32(1): 1-4.

Dasgupta, S. (1968). Relative predictability of five indices of adoption of recommended farm practices. Sociologia Ruralis, 8: 1-21.

Goswami, A. (2000). The Impact of Extension Education on the Social, Psychological and administrative behaviour of the livestock owners of the Sundarbans, West Bengal. Ph.D. thesis submitted to the University of Kalyani, West Bengal.

Goswami, M.(2007). A study on adoption behaviour of Kroiler poultry farmers in Murshidabad district of West Bengal. M.V.Sc. thesis submitted to W.B. University. of Animal \& fishery Scs.

Hazarika, P. (1983). A study of correlates of knowledge and adoption behaviour of the dairy farmers under I.C.D.P., Khanapara, Assam. M.Sc. Thesis, Kurukshetra University, Kurukshetra.

Hussain, M. A. (1968). Adoption of improved animal husbandry practices in Hyderabad district. M.Sc. Thesis submitted to Andhra Pradesh Agriculture University, Hyderabad.

Nataraju, M. S. and Channegowda, M. B. (1984). Differential adoption of improved dairy practices by small \& marginal farmers \& agril laborers. Indian Journal of Extn Edn, 20(3\&4): 63.

Roy, R. (2006). Adoption behaviour of Kroiler poultry farmers in Sundarban 
of West Bengal. M. V. Sc. thesis submitted to W.B. University. of Animal \& fishery Sciences.

Singh, J. N. (1982). A study of suitability of dairy farming innovations and factors associated with their adoption by cattle owners of ICDP, Gurgaon (Haryana). Ph.D. Thesis submitted to Kurukshetra University, Kurukshetra.

Sundaraswami, B.; Rao, M. K. S. and Awatigar, M. B. (1978). Impact of T.V. on farmers' knowledge. Indian Journal of Extension Education, 39(1): 23-26.

Tripathi, S. L. and Jyoti, J. S. (1971). Sociopersonal factors influencing the adoption of improved farm practices. Allahabad Farmer, 18(5): 347-354.

Jha, P. N. and Shaktwat, G. S. (1972). Adoption behavior of farmers towards hybrid Bajra cultivation. Indian Journal of Extension Education, 7(1 \&2): 24-29.

Kunzru, O. N.; Sagar, R. L. and Srivastava, N. K. (1987). Constraints in adoption of HS vaccine. Indian Journal of
Extension Education, 23(3 \&4): 74-76.

Sarkar, A. K. (1981). A study of communication patterns and factors affecting adoption of technology in West Bengal. Ph.D. Thesis submitted to National Dairy Research Institute, Karnal.

Sayeedi, A. G. (1983). A study of knowledge, attitude and adoption of dairy farmers towards dairy scientific activities in Jammu District (J \& K State). A dissertation, submitted to Kurukshetra University, Kurukshetra.

Sivanarayana, G. and Jayarama, Reddy, S. (1995). Constraints in the adoption of improved sheep and goat practices by the small and marginal farmers of diversified farming. Indian Journal of Dairy Science, 48(4): 306-308.

Sohal, T. S.; Singh, J. N. and Singh, J. P. (1982). Impact of extensive extension education on the adoption of scientific dairy farming by landless rural families around Karnal (Haryana). Indian Journal of Dairy Science, 35(4): 447-453.

\section{How to cite this article:}

Arunasis Goswami and Sukanta Biswas. 2020. Knowledge based Adoption Behavior of Poultry Farmers in Different Agro-Climatic Zones of West Bengal, India. Int.J.Curr.Microbiol.App.Sci. 9(12): 2525-2534. doi: https://doi.org/10.20546/ijcmas.2020.912.300 